BMJ Open Gastroenterology

\title{
Clinical and genetic profile of patients with seronegative coeliac disease: the natural history and response to gluten-free diet
}

\author{
Maria Pina Dore, ${ }^{1},{ }^{2}$ Giovanni Mario Pes, ${ }^{1}$ Ivana Dettori, ${ }^{1}$ Vincenzo Villanacci, ${ }^{3}$ \\ Alessandra Manca, ${ }^{4}$ Giuseppe Realdi ${ }^{1}$
}

\section{ABSTRACT}

Objectives: Patients with clinical, genetic and histological features of coeliac disease (CD), but negative for serological markers, pose a significant clinical problem. The aim of this study was to outline a specific profile, and to evaluate the natural history and response to gluten-free diet (GFD) of patients with seronegative $\mathrm{CD}$.

Methods: patients with duodenal mucosa damage Marsh I, II and III stages, HLA DQ2/DQ8 haplotype and clinical features suggestive of $C D$, but negative for $C D$ serology, were defined as seronegative $C D$ patients. Other common causes of duodenal mucosa damage were excluded. HLA-DR and DQ genotype/haplotype between all Marsh stages of patients with seronegative and seropositive $C D$ were compared. Clinical features, laboratory testing and histological findings were evaluated after a GFD and a gluten rechallenge. A long follow-up period was available.

Results: 48 patients fulfilled diagnostic criteria over a 4-year period. Clinical phenotype and HLA-DR and $D Q$ frequencies between patients with seronegative and seropositive CD was similar. However, Marsh I stage was more prevalent in seronegative patients ( $42 \%$ vs $22 \%$; $p<0.05$ ). After a 1 -year GFD trial, clinical symptoms, histological features and laboratory testing improved in 40 patients and worsened in those who underwent a 6 -months gluten challenge. Five patients with seronegative CD $(25 \%)$ experienced the occurrence of autoimmune diseases during a median follow-up of 133 months (range 72-192).

Conclusions: Patients with seronegative $C D$ did not display a specific profile. They benefitted from GFD as patients with seropositive $C D$. Waiting for more sensitive serological markers, the diagnosis of seronegative $\mathrm{CD}$ remains a diagnosis of exclusion. of Brescia, Brescia, Italy

${ }^{4}$ Pathology Section,

Department of Clinical and Experimental Medicine, University of Sassari, Sassari, Italy

\section{Correspondence to} Professor Maria Pina Dore; mpdore@uniss.it

\section{Summary box}

What is already known about this subject?

- Seronegative coeliac disease occurs in a small proportion of patients.

- The diagnosis and treatment of patients with seronegative coeliac disease pose a clinical problem.

What are the new findings?

- In patients with seronegative coeliac disease, homozygosity for HLA-DQ-B1*02 genotype is less frequent compared with patients with seropositive coeliac disease.

- Clinical, histological and laboratory parameters improved in patients with seronegative coeliac disease following a gluten-free diet trial.

- Natural history of patients with seronegative coeliac disease is similar to patients with seropositive coeliac disease.

How might it impact on clinical practice in the foreseeable future?

- Waiting for more sensitive serological markers, the diagnosis of seronegative coeliac disease remains a diagnosis of exclusion.

- A long-term gluten-free diet is still the best treatment option in patients with seronegative coeliac disease.

in gluten, in genetically predisposed individuals. ${ }^{12}$ More specifically, CD occurs in carriers of HLA-DQ2 and/or DQ8 alleles, although HLA-unlinked loci may also contribute to the genetic susceptibility of the disease. ${ }^{3}$ Interactions between environmental and genetic factors are essential to trigger the immunological cascade leading to a complete loss of villi and nutrient malabsorption as a consequence. ${ }^{45}$

The first approach to diagnose CD is noninvasive. ${ }^{67}$ The preferred test is based on serology while patients are on a free diet. IgA anti-tissue transglutaminase (TTG) antibody 
is the most recommended test given its high accuracy. In patients with increased risk to develop CD, IgGdeamidated gliadin peptides (DGPs) may also be tested. Baseline IgA endomysial antibody (EMA) should be assessed in patients on a gluten-free diet (GFD). ${ }^{7}$ Patients with positive serologic tests should undergo an upper endoscopy with small-bowel biopsy to confirm the diagnosis of $\mathrm{CD}$. The diagnosis is presumptively established when there is concordance between the serologic and the biopsy findings. However, it is definitely confirmed when symptoms improve following gluten avoidance. ${ }^{8}$ Demonstration of histological normalisation is not always required. In clinical practice, there are occasionally individuals with negative serologic tests despite the presence of features suggesting CD. These patients should be tested for HLA-DQ2 (DQA1*05; DQB1*02) and/or DQ8 (DQA1*03; DQB1*0302) haplotypes because $\mathrm{CD}$ is highly unlikely to occur in patients lacking these haplotypes. ${ }^{9}$ In positive patients, smallbowel biopsies are needed to confirm the diagnosis. Histologic findings in the duodenal mucosa are usually described using the Marsh-Oberhuber classifications. ${ }^{10} 11$ They may be characterised by solely a mild increase of intraepithelial lymphocytes (IEL), or enhanced apoptosis and crypt hyperplasia, up to a complete loss of villi and total mucosal atrophy. ${ }^{10} 11$ However, lymphocytic infiltration of the intestinal epithelium and villous atrophy are not specific for CD and may be present in a variety of other disorders such as small-intestinal bacterial overgrowth, Crohn disease, giardiasis and a number of other less frequent causes. ${ }^{12}$ Demonstration of histological and laboratory tests improvement and/or normalisation is always required after 6-24 months of a GFD, because a subgroup of patients with irritable bowel syndrome may have symptomatic benefit from a GFD. ${ }^{13}$ Owing to this phenomenon, some authorities recommend to repeat duodenal biopsy after a gluten rechallenge, although this approach remains uncertain, and more importantly, quite unpleasant for patients.

In this study, patients with different Marsh stages with clinical, histological and genetic features suggestive of CD but negative for serological markers were investigated and compared with patients with seropositive CD. In addition, natural history and response to a GFD and gluten rechallenge were evaluated.

\section{METHODS}

\section{Study design}

This was a prospective cohort study. In the first step, patients with a 'presumptive' diagnosis of CD based on clinical features, HLA-DQ2 and/ DQ8 alleles and smallintestine histology, but negative for serology were enrolled in the study. Although the major Gastroenterological Societies $^{2}$ define seronegative CD patients as those with villus atrophy, in order to make easier for readers, in our study the definition of 'seronegative' $\mathrm{CD}$ also included patients with Marsh I and II stages. Phenotype and
HLA-DR and DQ haplotypes of patients with seronegative CD were compared with patients with seropositive CD. Seronegative patients were invited to undergo a GFD for 12 months followed by a second endoscopy with duodenal biopsy, laboratory testing and clinical assessment. In the third step, patients with seronegative $\mathrm{CD}$ were checked by an additional duodenal biopsy at the end of a full gluten challenge. A follow-up of patients with seronegative $\mathrm{CD}$ was also available.

\section{Setting}

Patients attending the Coeliac Disease Centre at the Clinica Medica, University of Sassari, Italy, from 2000 to December 2004, were evaluated for this study. The Clinica Medica hospital is a tertiary referral centre that provides care for patients with gastrointestinal problems including most adult cases of CD in Northern Sardinia. The population of this region is entirely white and one of the most genetically homogeneous in the world. ${ }^{14}$

\section{Inclusion criteria}

Patients with $\mathrm{CD}$ on the basis of clinical features, proximal small-intestinal biopsies compatible with CD according to Marsh classification and positive for HLA-DQ2 and/or DQ8 alleles but negative for serology markers were enrolled in the study. Written informed consent was obtained from each patient. The protocol was approved by the local ethics committee, Azienda Unità Sanitaria Locale N 1, Sassari, Italy.

\section{Exclusion criteria}

Patients with Helicobacter pylori infection, ova and parasites such as giardiasis and/or other intestinal infections in the stool, Crohn disease, small-intestinal bacterial overgrowth, drug-induced enteropathy and established diseases including liver and pancreas disease or cancer, and eosinophilic infiltrate in duodenal specimens were excluded from the study.

\section{Control group}

Patients with a new diagnosis of seropositive CD were evaluated in the same fashion as that of patients with seronegative CD. Clinical features, laboratory testing and genotyping were compared between patients with seropositive and seronegative CD.

\section{Patient work-up}

Before enrolment, patients underwent a diagnostic approach as follows:

1. History and clinical examination.

2. Laboratory tests: complete blood count, erythrocyte sedimentation rate, $\mathrm{C}$ reactive protein, protein electrophoresis, $B_{12}$ vitamin and folic acid levels, ferritin, iron, calcium, thyroid hormones, serum immunoglobulins, albumin, triglyceride, cholesterol, liver and pancreas function tests and glycaemic levels.

3. Stool samples were examined for ova, bacteria and parasites. 
4. Serology markers for CD: IgA and IgG antibodies for native gliadin (AGA), EMA and TTG.

5. Breath tests (BTs): the presence of $H$. pylori infection was confirmed by ${ }^{13} \mathrm{C}$-urea BT (UBT). Lactose malabsorption and small-intestinal bacterial overgrowth were assessed by conventional hydrogen BT.

6. Endoscopy: each patient underwent upper endoscopy. Tissue specimens were taken from the antrum, angulus, and from the corpus of the stomach. Three additional biopsies were obtained from the duodenum.

7. HLA-DQ2/DQ8 proteins.

\section{Diagnostic criteria}

Serology

Antibody testing was based on the identification of IgA and IgG TTG antibodies by a validated, standardised and reliable commercial kit (Eurospital, Trieste, Italy). IgA and IgG AGA antibodies were assessed by the immunocup system (Pharmacia \& Upjohn Diagnostic, Uppsala, Sweden) and IgA EMA antibodies were detected by indirect immunofluorescence on monkey oesophagus (ALPHADIA sa/nv, Diagnostic Products, Wavre, Belgium).

\section{Breath tests}

The presence of $H$. pylori infection was confirmed by ${ }^{13}$ C-urea BT (AB Analitica Srl, PD, Italy). ${ }^{15}$ Lactase status was evaluated using the conventional hydrogen $\mathrm{BT}$ after ingestion of $25 \mathrm{~g}$ of lactose. Small-intestinal bacterial overgrowth was assessed by hydrogen BT after administering a dose of $50 \mathrm{~g}$ of glucose. ${ }^{16}$

\section{Histology}

Mucosal sections were evaluated by two expert gastrointestinal pathologists ( $\mathrm{VV}$ and $\mathrm{AM}$ ) independently. In the case of CD histologic features, the Marsh classification was applied. Briefly, a Marsh I stage corresponded to a cut-off of $>25$ IEL per 100 enterocytes, increased IEL associated with crypt hyperplasia was classified as Marsh II, and partial, subtotal or total villi atrophy as Marsh IIIa, IIIb and IIIc, respectively. The presence of CD3 lymphocytes was also confirmed by immunostaining.

\section{HLA-DQ2/DQ8 proteins}

The presence of HLA-DQ2/DQ8 was assessed by an ELISA commercial kit (Eurospital, Trieste, Italy).

\section{Genotyping}

DNA was extracted using the Chelex method. PCR amplification of the polymorphic second exon of the HLA-DRB1, DQB1 genes and dot-blot analysis of amplified DNA with sequence-specific oligonucleotide (SSO) probes were carried out as previously reported. ${ }^{17}$

\section{Statistical analysis}

Mann-Whitney $\mathrm{U}$ test was used to compare the age of seronegative versus seropositive CD patients at diagnosis, as well as the other continuous variables. Pearson $\chi^{2}$ test was used to compare the categorical variables between the patient subgroups. For histological analysis, the Marsh IIIa, IIIb and IIIc categories were merged together. ${ }^{18}$ Similarly, the presence of osteoporosis and osteopenia was recoded into a single category.

The association of the Class II HLA-DR-DQ haplotypes with $\mathrm{CD}$ was assessed with data points arranged in contingency tables and assessed by the Fisher's exact test. Haplotypes were grouped for $\mathrm{DQ}_{2}$ according to their association with DR3 haplotype or with DR5/DR7 haplotypes. DQ8, encoded by DQB $1 * 0302$ allele, is generally associated to DR4 haplotype.

All tests were performed as two-tailed and differences were considered statistically significant when $\mathrm{p}<0.05$. Statistical analysis was carried out using SPSS V.16.0 software (SPSS Institute, Illinois, USA).

\section{RESULTS}

\section{First step}

A total of 48 patients with a median age of 36 years; range 16-76, were included in the study (40 female patients; F/M 5:1). All cases were negative for CD serology markers (except four for AGA IgG and one for AGA IgA) and positive for histological alterations in the small-intestinal mucosa according to Marsh classification, CD3 immunostaining and HLA DQ2/DQ8 alleles. Patients were tested for bone mineral density and invited to undergo a GFD for 12 months (figure 1).

First step

48 Seronegative $C D$ patients enrolled

(Positive for HLA DQ2/DQ8, Histology and Clinical features

Negative for diseases associated with duodenal villus atrophy)

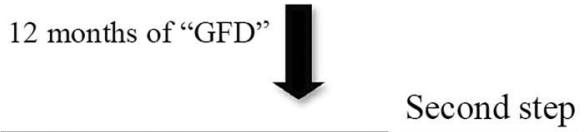

40 Seronegative CD patients repeated

EGDS plus duodenal biopsies

Clinical examination

Laboratory testing

6 months of gluten challange

Third step

11 Seronegative $C D$ patients repeated

EGDS plus duodenal biopsies

Clinical examination

Laboratory testing

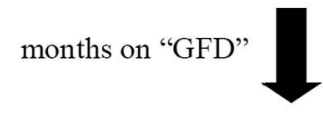

Follow-up

20 Seronegative $\mathrm{CD}$ patients followed up

Figure 1 Flow diagram of the patients with seronegative coeliac disease prospectively studied according to each step. 


\section{Patient features}

Clinical features of patients with seronegative CD are shown in table 1. Abdominal pain lasting for at least 1 year, in the majority of cases associated with abdominal bloating, was the most common symptom (44/48; 92\%). Laboratory tests were altered in most patients (table 1). Notably, low IgA levels were not detected in any patient.

A high proportion of patients $(63 \%)$ displayed lactose malabsorption; however, it was not considered to be the sole cause of intestinal injury. Autoimmune disorders were associated with seronegative CD only in few cases (type I diabetes in one patient, Hashimoto's thyroiditis in five patients). In six patients, a non-herpetiformis dermatitis was observed. Use of angiotensin II inhibitors, nowadays known to be harmful for intestinal mucosa, ${ }^{16}$ was not recorded in any of the 48 studied patients with the exception of one 67-year-old female patient, who was taking enalapril.

Table 1 Clinical features of 85 patients with seropositive coeliac disease and 48 patients with seronegative coeliac disease at the baseline and after 1 year of gluten-free diet

\begin{tabular}{|c|c|c|c|}
\hline Features & $\begin{array}{l}\text { Seropositive } \\
\text { coeliac } \\
\text { disease } \\
\end{array}$ & $\begin{array}{l}\text { Seronegative } \\
\text { coeliac disease, } \\
\text { basal (free diet) }\end{array}$ & $\begin{array}{l}\text { Seronegative coeliac } \\
\text { disease, after } 1 \text { year } \\
\text { of gluten-free diet }\end{array}$ \\
\hline No. of patients & 85 & 48 & 40 \\
\hline Men/women & $16 / 69$ & $8 / 40$ & $6 / 34$ \\
\hline Median age (range), years & $35(16-74)$ & $36(18-76)$ & $36(22-69)$ \\
\hline \multicolumn{4}{|l|}{ Clinical signs and symptoms } \\
\hline Abdominal pain & $65(76 \%)$ & $44(92 \%)$ & $4(10 \%)^{\star *}$ \\
\hline Diarrhoea & $62(73 \%)$ & $36(75 \%)$ & $3(7 \%)^{* *}$ \\
\hline Alternating bowel movements & $21(25 \%)$ & $8(17 \%)$ & $0(0 \%)^{\star \star}$ \\
\hline Familiarity for coeliac disease & 32 (37\%) & $8(17 \%)^{*}$ & - \\
\hline \multicolumn{4}{|l|}{ Laboratory tests } \\
\hline Haemoglobin & $12.4 \pm 1.6$ & $12.7 \pm 1.3$ & - \\
\hline Haemoglobin $<12$ mg/dL & $45(53 \%)$ & $5(10 \%)^{\star *}$ & $0(0.0 \%)^{*}$ \\
\hline Mean corpuscular volume $<80 \mathrm{fL}$ & - & $6(12 \%)$ & $1(2.0 \%)^{*}$ \\
\hline Mean corpuscular haemoglobin $<27 \mathrm{ng} / \mathrm{mL}$ & - & $4(8 \%)$ & $0(0.0 \%)$ \\
\hline Lipase $>30 \mathrm{U} / \mathrm{L}$ & $83(86 \%)$ & $3(6 \%)^{\star \star}$ & $0(0.0 \%)$ \\
\hline Amylase $>120 \mathrm{U} / \mathrm{L}$ & $3(3 \%)$ & $4(8 \%)$ & $0(0.0 \%)$ \\
\hline AST/ALT & $6(7 \%)$ & $3(6 \%)$ & $1(2.0 \%)$ \\
\hline Ferritin mg/dL & $30.0 \pm 37.0$ & $17.5-24.1$ & - \\
\hline Ferritin $<50 \mathrm{mg} / \mathrm{dL}$ & $68(80 \%)$ & $11(23 \%)^{\star \star}$ & $8(20 \%)$ \\
\hline Vitamin $\mathrm{B}_{12}$ & $389.1 \pm 203.7$ & $285.2 \pm 282.4$ & $324.1 \pm 180.7$ \\
\hline Vitamin $B_{12}<200 \mathrm{ng} / \mathrm{mL}$ & $13(15 \%)$ & $3(6 \%)$ & $0(0.0 \%)$ \\
\hline Folate $<7 \mathrm{ng} / \mathrm{mL}$ & 32 (38\%) & $7(15 \%)$ & $1(2.0 \%)^{*}$ \\
\hline Calcium $<9 \mathrm{mg} / \mathrm{dL}$ & - & $0(0 \%)$ & $0(0.0 \%)$ \\
\hline Blood glucose, $\mathrm{mg} / \mathrm{dL}$ & $88.2 \pm 9.9$ & - & - \\
\hline Blood glucose $<100 \mathrm{mg} / \mathrm{dL}$ & - & $1(2 \%)$ & $0(0.0 \%)$ \\
\hline Albumin, $g / L$ & $53.7 \pm 4.1$ & $46.3 \pm 19.1$ & - \\
\hline Albumin $<35 \mathrm{~g} / \mathrm{L}$ & $0(0 \%)$ & $3(6 \%)$ & - \\
\hline Total cholesterol, mg/dL & $171.1 \pm 32.2$ & $169.4 \pm 34.3$ & - \\
\hline Total cholesterol $<200 \mathrm{mg} / / \mathrm{dL}$ & $69(81 \%))$ & $37(77 \%)$ & - \\
\hline Triglycerides, mg/dL & $65.1 \pm 34.2$ & $66.5 \pm 35.9$ & - \\
\hline Triglycerides <150 mg/dL & $83(97 \%)$ & $45(94 \%)$ & - \\
\hline \multicolumn{4}{|l|}{ Breath testing } \\
\hline Lactose malabsorption & 77 (91\%) & $30(63 \%)$ & - \\
\hline \multicolumn{4}{|l|}{ Histology } \\
\hline Marsh 0 & $0(0 \%)$ & $0(0 \%)$ & $13(33 \%)^{\star \star}$ \\
\hline Marsh I & $19(22 \%)$ & $20(42 \%)^{*}$ & $25(63 \%)$ \\
\hline Marsh II & $28(33 \%)$ & $13(27 \%)$ & $1(2 \%)^{\star *}$ \\
\hline Marsh III & $38(45 \%)$ & $15(31 \%)$ & $1(2 \%)^{\star \star}$ \\
\hline \multicolumn{4}{|l|}{ Comorbidities } \\
\hline Dermatitis & $15(18 \%)$ & $6(12.5 \%)$ & $0(0 \%)$ \\
\hline Diabetes & $3(3 \%)$ & $1(2 \%)$ & $1(2.5 \%)$ \\
\hline Thyroiditis & $14(16 \%)$ & $5(10 \%)$ & $5(10 \%)$ \\
\hline Osteopenia/osteoporosis & 29 (34\%) & $21(44 \%)$ & $5(12.5 \%)$ \\
\hline Mild depression & $8(9 \%)$ & $13(27 \%)$ & $3(6 \%)$ \\
\hline
\end{tabular}


Histology

According to the Marsh classification, the most observed lesion in the duodenal mucosa of patients with seronegative CD was Marsh I (20/48; 42\%). Surprisingly, 15 patients displayed Marsh III (table 1).

\section{Seronegative and seropositive patients}

Patients with seronegative CD were compared with 85 patients with seropositive CD (table 1). Median age and gender ratio ( $\mathrm{F} / \mathrm{M} 5: 1$ vs $\mathrm{F} / \mathrm{M}$ 4:1) between the two $\mathrm{CD}$ groups were similar. Reported abdominal symptoms were more frequent in seronegative CD $(92 \%$ vs $76 \%$, $\mathrm{p}=0.029$ ), whereas family history of CD was more frequent among patients with seropositive $\mathrm{CD}$ (37\% vs $17 \%, \mathrm{p}=0.011)$. Overall, patients with seronegative CD did not display a specific laboratory profile, moreover alterations of biochemical tests were, in general, less common than in patients with seropositive CD. In addition, severe duodenal mucosal damage (Marsh II-III) was observed less frequently in patients with seronegative CD compared with patients with seropositive CD $(58 \%$ vs $78 \%, \mathrm{p}=0.019$ ) (table 1). This may explain the lower frequency of lactose malabsorption in patients with seronegative CD compared with patients with seropositive CD (63\% vs 91\%, p=0.0001). Autoimmune diseases such as diabetes type 1 and Hashimoto's thyroiditis and an established mild anxiety-depression syndrome as well as non-herpetiformis dermatitis were more often associated with patients with seronegative CD. Instead, osteopenia and/or osteoporosis were more frequent in patients with seronegative CD than in patients with seropositive CD. Additional analyses were performed in order to compare Marsh II and III stages of seronegative CD patients with seropositive CD patients (see online supplementary material).

\section{Genotyping}

Class II HLA haplotypes were assessed in 32 patients with seronegative $\mathrm{CD}$ and in 62 patients with seropositive CD. Findings were also compared with the Sardinian general population. ${ }^{17}$ The frequency of HLA-DR and
DQ haplotypes is presented in table 2. As expected, almost half of seropositive patients were homozygous for HLA-DR3-DQB1*02 and HLA-DR4-DQB1*0302 haplotypes which are strongly predisposing for $\mathrm{CD}$. On the contrary, among seronegative patients, the frequency did not differ significantly from that of the general population. Moreover, the frequency of homozygotes DQB $1 * 02$, usually associated with a more severe disease, was twofold higher in patients with seropositive CD (38.1\% vs $15.2 \%$ ) compared with seronegative CD (data not shown). Notably, none of the seronegative patients was negative for alleles predisposing to $\mathrm{CD}$.

\section{Second step}

After 1 year of GFD, patients were interviewed for diet compliance and symptoms. A straight adherence to GFD was recorded for 44 patients (92\%). Four patients reported difficulties to completely adhere to the GFD. All patients were invited to repeat the esophago-gastroduodenoscopy (EGDS) with duodenal biopsy and laboratory testing (figure 1). Among the 48, 40 patients agreed, 34 were females; median age 36 years (range 22-69) (table 1). There was a dramatic improvement of abdominal symptoms that was statistically significant among patients with seronegative CD that completely adhere to GFD. In addition, haemoglobin levels gained normalisation in all five patients and folate was in the reference range in six patients out of seven (86\%) (table 1). More interesting, Marsh III mucosal duodenal injury resolved in 14 patients out of 15 (93\%), out of 40 undergoing EGDS, and a complete restitutio ad integrum was observed in 13 patients (table 1). Bone mineral density significantly increased in the lumbar spine and femoral neck compared with previous examination (table 1).

\section{Third step}

Patients with seronegative $\mathrm{CD}$ on GFD for up to 12 months and re-evaluated for duodenal mucosa features were invited to undergo a gluten challenge (figure 1). Among 40 patients, 19 agreed to follow a gluten-rich diet trial. However, eight patients avoided food

Table 2 Distribution of HLA-DR and DQ haplotypes in patients with seropositive and seronegative coeliac disease and in a population of 631 controls from Sardinia ${ }^{17}$

\begin{tabular}{|c|c|c|c|c|}
\hline \multirow[b]{2}{*}{ DRB1 } & \multirow[b]{2}{*}{ DQB1 } & \multicolumn{2}{|c|}{ Patients with coeliac disease } & \multirow{2}{*}{$\begin{array}{l}\text { Population controls } \\
631 \text { subjects }\end{array}$} \\
\hline & & 62 seropositive patients & 32 seronegative patients & \\
\hline 0301 & 0201 & $65(52.4 \%)$ & $18(28.1 \%)$ & $277(21.9 \%)$ \\
\hline 04 & 0302 & $14(11.3 \%)$ & $8(12.5 \%)$ & $94(7.4 \%)$ \\
\hline 04 & 0301 & $2(1.6 \%)$ & $2(3.1 \%)$ & $41(3.2 \%)$ \\
\hline 04 & 0201 & $3(2.4 \%)$ & $4(6.3 \%)$ & $16(1.3 \%)$ \\
\hline 0701 & 0201 & $12(9.7 \%)$ & $2(3.1 \%)$ & $72(5.7 \%)$ \\
\hline $11,12,13$ & 0301 & $10(8.1 \%)$ & $7(10.9 \%)$ & $209(16.6 \%)$ \\
\hline 16 & 0502 & $9(7.3 \%)$ & $10(15.6 \%)$ & $241(19.1 \%)$ \\
\hline Others & & $9(7.3 \%)$ & $13(20.3 \%)$ & $312(24.7 \%)$ \\
\hline Total & & 124 & 64 & 1262 \\
\hline
\end{tabular}


containing gluten after few weeks because of the occurrence of abdominal symptoms. All 11 patients underwent a third EGDS with duodenal biopsies after 6 months of free diet. Examination of duodenal specimens according to Marsh classification showed no patient with Marsh 0, three patients with Marsh I, eight patients with Marsh II and no one with Marsh 3, respectively (table 3). All patients reported recurrence of gastrointestinal symptoms.

\section{Follow-up}

Twenty patients were available for the follow-up after a median of 133 months (range 72-192 months) (table 4). The majority $(90 \%)$ were female patients. Three of them developed autoimmune thyroiditis, and inflammatory bowel disease, ulcerative colitis and Crohn disease, respectively, occurred in two patients.

\section{DISCUSSION}

In clinical practice, $\mathrm{CD}$ negative for serological markers is a problem for the physician and the patient. First of all, to differentiate between seronegative $\mathrm{CD}$ and other causes of duodenal mucosal injury needs an extensive work-up; second, treatment and follow-up of patients with seronegative $\mathrm{CD}$ pose a clinical dilemma. ${ }^{19}$

Over a 4-year period, among patients with gastrointestinal symptoms seen at our single tertiary-care referral centre, a definitive diagnosis of seronegative $\mathrm{CD}$ was established in 48. An interesting finding in our cohort was the high number of patients who improved their symptoms after a period of GFD and, more importantly, the restitutio ad integrum of intestinal mucosa observed in the majority of patients in the duodenal specimens obtained at the second upper endoscopy. Notably, patients with seronegative CD with stigmata of malabsorption experienced normalisation of laboratory parameters, especially haemoglobin and folate serum levels,

Table 3 Comparison of duodenal mucosa findings according to Marsh classification in patients with seronegative coeliac disease after 1 year of gluten-free diet followed by a gluten challenge

\begin{tabular}{lcccc}
$\begin{array}{l}\text { Patient } \\
\text { number }\end{array}$ & Gender & Age & \multicolumn{2}{c}{ Marsh classification } \\
\cline { 3 - 5 } 1 & M & 31 & 0 & Marsh GCD \\
2 & F & 46 & 0 & II \\
3 & F & 42 & 0 & II \\
4 & F & 44 & 0 & II \\
5 & M & 41 & I & II \\
6 & M & 26 & 0 & II \\
7 & F & 47 & 0 & II \\
8 & F & 15 & 0 & I \\
9 & F & 29 & I & II \\
10 & F & 34 & I & II \\
11 & F & 20 & 0 & I \\
\hline GC, gluten challenge; GFD, gluten-free diet.
\end{tabular}

according to restoration of the intestinal villi following GFD. These findings support our initial choice to recommend a GFD trial in patients with seronegative CD, although the benefit of a lifelong GFD in order to prevent autoimmune diseases, usually associated with $\mathrm{CD}$, is not so obvious according to our findings. In fact, in our patients with seronegative $\mathrm{CD}$ followed up for several months (133), the occurrence of autoimmune diseases was observed in $25 \%$ who were given the GFD. However, these results can be partially explained by the fact that genes predisposing to $\mathrm{CD}$ are also involved in other autoimmune diseases such as thyroiditis and diabetes, both diseases in Sardinia have one of the highest prevalence in Europe. ${ }^{2021}$

At the moment, we do not have an explanation for negative serum markers in CD. Usually, TTG IgA and endomysial IgA are highly sensitive and specific for $\mathrm{CD}$, whereas anti-gliadin antibody testing is not. ${ }^{21-23}$ Some authors explain seronegativity as the inability of anti-TTG passage into the bloodstream. ${ }^{24-26}$ For example, in seronegative $\mathrm{CD}$ children with Marsh I stage, Tosco $e t a l^{25}$ found deposits of TTG bound with IgA in the duodenal mucosa by immunofluorescence. An additional study observed a clinical improvement following a GFD in patients with increased presence of IELs in the duodenal mucosa and in ten of them deposits of immunocomplexes. ${ }^{26}$

The newer DGP assay is also highly sensitive and specific for $\mathrm{CD}$ and has been shown to detect patients who were seronegative by TTG testing; ${ }^{27}{ }^{28}$ however, at the time of patient enrolment antibodies to DGP were not yet available. In addition, it has been reported that CD markers may be negative in the presence of partial villus atrophy such as in subclinical or silent $\mathrm{CD} .{ }^{29}$ This could justify negative $\mathrm{CD}$ markers in the 20 patients with mild intestinal mucosa damage (Marsh I), but is inexplicable for the 28 patients with Marsh II and III. We may also cautiously suppose that in seronegative CD patients autoantibodies might have disappeared in adulthood, similarly to other autoimmune diseases such as type 1 diabetes. ${ }^{30}$ An additional issue may be that until 2012, the antihypertensive drug olmesartan was not recognised as a potential factor of severe villus atrophy; ${ }^{16}$ however, in our cohort of young patients, hypertension was not recorded among comorbidities except for one 47-year-old woman taking enalapril.

The existence of seronegative $\mathrm{CD}$ was previously reported in several studies, ${ }^{31-35}$ although its prevalence is still a matter of debate and available information on the clinical phenotype of this subset of patients with $\mathrm{CD}$ is currently discordant. For example, Rostami $e t a l^{31}$ reported a high prevalence of seronegative CD in 1999. In this study, patients had mild intestinal alterations and moderate gastrointestinal symptoms. Abrams and colleagues reported a prevalence of patients negative for all CD-related serological tests in $15 \%$ of their patients with villus atrophy. ${ }^{32}$ The most striking finding of their study was that clinical features and associated 
Table 4 Comorbidity developed by 20 patients with seronegative coeliac disease during the follow-up period (median=133 months; range 72-192 months)

\begin{tabular}{llll}
\hline Patient number & Months of follow-up & Genderlage & Comorbidity developed during follow-up \\
\hline 1 & 182 & $F / 56$ & Major depression, diverticulosis \\
2 & 142 & $\mathrm{M} / 55$ & Osteopenia \\
3 & 131 & $\mathrm{~F} / 58$ & Osteoporosis, thyroiditis, arthralgia, reflux disease \\
4 & 135 & $\mathrm{~F} / 43$ & Gestational diabetes \\
5 & 120 & $\mathrm{~F} / 83$ & As before \\
6 & 84 & $\mathrm{~F} / 50$ & Angioedema, urticaria, C1 deficiency, asthma \\
7 & 96 & $\mathrm{~F} / 63$ & Thyroiditis \\
8 & 83 & $\mathrm{~F} / 41$ & None \\
9 & 128 & $\mathrm{~F} / 58$ & As before \\
10 & 72 & $\mathrm{~F} / 39$ & Kidney stone \\
11 & 144 & $\mathrm{~F} / 61$ & Hypercholesterolemia and obesity \\
12 & 158 & $\mathrm{~F} / 35$ & Irritable bowel syndrome with constipation \\
13 & 168 & $\mathrm{~F} / 61$ & Depression and osteoporosis \\
14 & 144 & $\mathrm{~F} / 36$ & Constipation \\
15 & 108 & $\mathrm{~F} / 50$ & Thyroiditis \\
16 & 121 & $\mathrm{~F} / 70$ & Chronic migraine headache \\
17 & 156 & $\mathrm{~F} / 43$ & Osteoporosis, breast cancer \\
18 & 72 & $\mathrm{~F} / 50$ & Ulcerative colitis \\
19 & 192 & $\mathrm{M} / 51$ & Crohn, melanoma and major depression \\
20 & 177 & $F / 49$ & Psoriasis \\
\hline
\end{tabular}

disease in patients with seronegative $\mathrm{CD}$ were similar to those with seropositive $\mathrm{CD}$ and improved following a GFD. ${ }^{32}$ In contrast, in a more recent study of adult patients with seronegative $\mathrm{CD}$, on the basis of negative TTG, DGP and EMA tests, positive genetic tests and histological findings consistent with a diagnosis of $\mathrm{CD}$, only two patients out of eight showed an initial improvement of symptoms after a GFD. ${ }^{34}$ In our study, the majority of seronegative patients experienced a reduction of symptoms on GFD. The long-lasting effect of GFD observed in our cohort on gastrointestinal symptoms tend to exclude a mere placebo effect. ${ }^{13}$ In patients with malabsorption, duodenal biopsy is needed in order to analyse the intestinal mucosa for villus atrophy. ${ }^{32}$ Although malabsorption was not detected in the $100 \%$ of our patients with seronegative CD, all had some degree of mucosal duodenal injury and, more importantly, symptoms improvement was associated with normalisation of duodenal mucosa.

Overall, differences in clinical phenotype and genotype between patients with seronegative and seropositive $\mathrm{CD}$ were minimal, insufficient to draw a specific profile. However, the frequency of DRB $1 * 02$ homozygosis, which entails a more severe underlying autoimmune process, was significantly reduced in seronegative patients, a possible indication that patients with seronegative CD have a relatively milder genetic profile.

There are limitations of our study. First of all, the long follow-up period and the invasive procedures in the different steps of the study determined a high number of dropouts. Moreover, patients were seen by different physicians over a 13-year follow-up period and some loss of data cannot be ruled out. Finally, a gluten challenge test was refused by several patients and for those who agreed, we were not able to guarantee for an adequate introduction of gluten per day.

\section{Conclusions}

Our study identified a subgroup of patients with clinical and genetic features associated with histological lesions peculiar of $\mathrm{CD}$ and negative serology that benefits from GFD. We failed to trace a specific phenotype and/or genotype in our cohort of patients with seronegative CD compared with patients with seropositive CD. Waiting for more sensitive serological markers, small-bowel biopsy remains the 'gold standard' for the diagnosis of gluten intolerance in patients with malabsorption and unexplained abdominal symptoms. A strict, lifelong gluten avoidance appears the most reasonable treatment for these patients.

Contributors MPD, GMP and GR were involved in acquisition of data, analysis and interpretation of data, drafting of the manuscript, study concept and design, and critical revision of the manuscript for important intellectual content. ID contributed to acquisition of data. VV and AM carried out analysis of biopsies and critically revised the manuscript for important intellectual content.

Competing interests None declared.

\section{Patient consent Obtained.}

Ethics approval The protocol was approved by the local ethics committee, Azienda Unità Sanitaria Locale N 1, Sassari, Italy.

Provenance and peer review Not commissioned; externally peer reviewed.

Data sharing statement No additional data are available.

Open Access This is an Open Access article distributed in accordance with the Creative Commons Attribution Non Commercial (CC BY-NC 4.0) license, which permits others to distribute, remix, adapt, build upon this work noncommercially, and license their derivative works on different terms, provided the original work is properly cited and the use is non-commercial. See: http:// creativecommons.org/licenses/by-nc/4.0/ 


\section{REFERENCES}

1. American Gastroenterological Association Medical Position Statement: Celiac Sprue. Gastroenterology 2001;120:1522-5.

2. AGA Institute Medical Position Statement on the Diagnosis and Management of Celiac Disease. AGA Institute. Gastroenterology 2006;131:1977-80.

3. Sollid LM, Thorsby E. HLA susceptibility genes in celiac disease: genetic mapping and role in pathogenesis. Gastroenterology 1993;105:910-22.

4. Kagnoff MF. Celiac disease. A gastrointestinal disease with environmental, genetic, and immunologic components. Gastroenterol Clin North Am 1992;21:405-25.

5. Schuppan D. Current concepts of celiac disease pathogenesis. Gastroenterology 2000;19:234-42.

6. Rubio-Tapia A, Hill ID, Kelly CP, et al. ACG clinical guidelines: diagnosis and management of celiac disease. Am J Gastroenterol 2013;108:656-76.

7. Leffler D, Schuppan D, Pallav K, et al. Kinetics of the histological, serological and symptomatic responses to gluten challenge in adults with coeliac disease. Gut 2013;62:996-1004.

8. Rubio-Tapia A, Rahim MW, See JA, et al. Mucosal recovery and mortality in adults with celiac disease after treatment with a gluten-free diet. Am J Gastroenterol 2010;105:1412-20.

9. Hadithi M, von Blomberg BM, Crusius JB, et al. Accuracy of serologic tests and HLA-DQ typing for diagnosing celiac disease. Ann Intern Med 2007;147:294-302.

10. Marsh MN, Crowe PT. Morphology of the mucosal lesion in gluten sensitivity. Baillieres Clin Gastroenterol 1995;9:273-93.

11. Oberhuber G, Granditsch G, Vogelsang $H$. The histopathology of coeliac disease: time for a standardized report scheme for pathologists. Eur J Gastroenterol Hepatol 1999;11:1185-94.

12. Malamut G, Cerf-Bensussan N, Cellier C. Identification of new cases of severe enteropathy has recently increased the spectrum of intestinal non-celiac villous atrophy. Expert Rev Gastroenterol Hepatol 2015;9:719-21.

13. Wahnschaffe U, Schulzke JD, Zeitz M, et al. Predictors of clinical response to gluten-free diet in patients diagnosed with diarrhea-predominant irritable bowel syndrome. Clin Gastroenterol Hepatol 2007;5:844-50.

14. Cavalli-Sforza LL, Menozzi P, Piazza A, et al. The history and geography of human genes. Princeton: Princeton University Press, 1994

15. Pironti A, Tadeu V, Pedroni A, et al. Role of routine small intestinal biopsy in adult patient with irritable bowel syndrome-like symptoms. Minerva Med 2010;10:129-34.

16. Rubio-Tapia A, Herman ML, Ludvigsson JF, et al. Severe spruelike enteropathy associated with olmesartan. Mayo Clin Proc 2012;87:732-8.

17. Lampis $\mathrm{R}$, Morelli $\mathrm{L}$, Congia $\mathrm{M}$, et al. The inter-regional distribution of HLA class II haplotypes indicates the suitability of the Sardinian population for case-control association studies in complex diseases. Hum Mol Genet 2000;9:2959-65.

18. N Marsh M, W Johnson M, Rostami K. Mucosal histopathology in celiac disease: a rebuttal of Oberhuber's sub-division of Marsh III. Gastroenterol Hepatol Bed Bench 2015;8:99-109.

19. Volta U, Caio G, Boschetti E, et al. Seronegative celiac disease: Shedding light on an obscure clinical entity. Dig Liver Dis 2016;48:1018-22.
20. Niegowska M, Paccagnini D, Burrai C, et al. Antibodies against proinsulin and homologous MAP epitopes are detectable in Hashimoto's thyroiditis Sardinian patients, an additional link of association. PLOS ONE 2015;10:e0133497.

21. Sardu C, Cocco E, Mereu A, et al. Population based study of 12 autoimmune diseases in Sardinia, Italy: prevalence and comorbidity. PLOS ONE 2012;7:e32487.

22. Rostom A, Dubé C, Cranney A, et al. The diagnostic accuracy of serologic tests for celiac disease: a systematic review. Gastroenterology 2005;128(Suppl 1):S38-46.

23. Hill ID. What are the sensitivity and specificity of serologic tests for celiac disease? Do sensitivity and specificity vary in different populations?. Gastroenterology 2005;128(Suppl 1):S25-32.

24. Ierardi E, Losurdo G, Piscitelli D, et al. Seronegative celiac disease: where is the specific setting? Gastroenterol Hepatol Bed Bench. 2015;8:110-6

25. Tosco A, Maglio M, Paparo F, et al. Immunoglobulin A anti tissue transglutaminase antibody deposits in the small intestinal mucosa of children with no villous atrophy. J Pediatr Gastroenterol Nutr 2008;47:293-8.

26. Kaukinen K, Peräaho M, Collin $P$, et al. Small-bowel mucosal transglutaminase 2-specific IgA deposits in coeliac disease without villous atrophy: a prospective and randomized clinical study. Scand $J$ Gastroenterol 2005;40:564-72.

27. Sugai E, Hwang $\mathrm{HJ}$, Vázquez $\mathrm{H}$, et al. New serology assays can detect gluten sensitivity among enteropathy patients seronegative for anti-tissue transglutaminase. Clin Chem 2010;56:661-5.

28. Villalta D, Alessio MG, Tampoia M, et al. Testing for lgG class antibodies in celiac disease patients with selective IgA deficiency. A comparison of the diagnostic accuracy of 9 IgG anti-tissue transglutaminase, $1 \mathrm{IgG}$ anti-gliadin and $1 \mathrm{IgG}$ anti-deaminated gliadin peptide antibody assays. Clin Chim Acta 2007;382:95-9.

29. Tursi A, Brandimarte G, Giorgetti G, et al. Low prevalence of antigliadin and anti-endomysium antibodies in subclinical/silent celiac disease. Am J Gastroenterol 2001:96:1507-10.

30. Turner R, Stratton I, Horton V, et al. UKPDS 25: autoantibodies to islet-cell cytoplasm and glutamic acid decarboxylase for prediction of insulin requirement in type 2 diabetes. UK Prospective Diabetes Study Group. Lancet 1997;350:1288-93.

31. Rostami K, Kerckhaert J, Tiemessen R, et al. Sensitivity of antiendomysium and antigliadin antibodies in untreated celiac disease: disappointing in clinical practice. Am J Gastroenterol 1999;94:888-94.

32. Abrams JA, Diamond $B$, Rotterdam $\mathrm{H}$, et al. Seronegative celiac disease: increased prevalence with lesser degrees of villous atrophy. Dig Dis Sci 2004;49:546-50.

33. Dahele A, Kingstone K, Bode J, et al. Antiendomysial antibody negative celiac disease: does additional serological testing help? Dig Dis Sci 2001;46:214-21.

34. Ashabani A, Errabtea $\mathrm{H}$, Shapan A, et al. Serologic markers of untreated celiac disease in Libyan children: antigliadin, antitransglutaminase, antiendomysial, and anticalreticulin antibodies. J Pediatr Gastroenterol Nutr 2001;33:276-82.

35. DeGaetani M, Tennyson CA, Lebwohl B, et al. Villous atrophy and negative celiac serology: a diagnostic and therapeutic dilemma. Am J Gastroenterol 2013;108:647-53. 